\title{
MAKING A ROOM FOR THE IOT: A DOMESTIC DEMONSTRATION
}

\author{
Joseph Lindley ${ }^{1}$, Adrian Gradinar ${ }^{1}$, Paul Coulton $^{1 *}$, Rachel Cooper ${ }^{1}$, Ian Forrester ${ }^{2}$ \\ ${ }^{1}$ Imagination Lancaster, Lancaster University, United Kingdom \\ ${ }^{2} B B C R \& D$ North lab, Mediacity UK, Salford, United Kingdom \\ *p.coulton@lancaster.ac.uk
}

Keywords: Object-Based Media, Perceptive Media, Immersion, Experiential Futures, Design Fiction

\begin{abstract}
This paper describes the process of constructing the 'Living Room of the Future' technology demonstrator. The demonstrator combines a mixture of commercial Internet of Things products with research prototypes in order to (a) demonstrate how the heterogenous IoT may augment media experiences in the home using 'Object-Based Media', (b) to contribute to the development of Experiential Design Fiction as a method for understanding issues relating to the 'Implications for Adoption' of emerging technologies, (c) to act as a research probe to explore the acceptability of IoT products and services in the home with respect to data and privacy.
\end{abstract}

\section{Introduction}

Among the array of opportunities the Internet of Things (IoT) offers, for media producers and broadcasters it may help deliver next-generation programmes that enable more immersive experiences. By utilising Object-Based Media (OBM) the sensing and actuating capabilities of domestic IoT devices can be utilised in such a way that programmes transcend the audio-visual and linear paradigms traditionally associated with broadcast to become 'Perceptive Media'. Combining IoT sensor data with pre-existing datasets allows Perceptive Media to dynamically adapt the programme content according to the individual viewer, viewing context, and the environment. For example, sound tracks may be dynamically adapted in order to maximise the affect in the viewer based on their taste. In addition to adapting screen-based content according to context IoT devices may be leveraged as 'actuators' to further augment a programme. By coding the structure of programmes semantically, OBM can, within the viewing environment, relate on-screen events to real-world events to subtly immerse the audience by utilising the ambient IoT devices. For example, smart lighting systems may be used to complement the cinematography, or heating/cooling systems to reflect on-screen climate in the real world. In this paper we describe the process of constructing a prototype that incorporates these abilities in a 'Living Room of the Future' (LRoTF) demonstrator in order to underpin three research contributions.

The paper's first contribution is a report of how LRoTF utilises $\mathrm{OBM}$ and IoT in order to create more immersive media experiences. The second contribution relates to the utilisation of Design Fiction as an approach to researching the plausible future impacts of adopting emerging technologies like the IoT. In the 10 years since Julian Bleecker popularised Design Fiction it has become a widely-applied technique for exploring possible socio-technical futures. The LRoTF builds upon prior work advocating Design Fiction as World Building as an approach to exploring the 'Implications for Adoption' relating to the IoT by adding an experiential element that literally situates audiences within the fictional world created by the prototype. The third contribution offers preliminary findings relating from the design, development, and installation of the LRoTF Experiential Design Fiction prototype. Reflecting on the process of building LRoTF we provide preliminary insights from the project relating the acceptability of domestic IoT devices when used in this context.

\section{Designing the Living Room of the Future}

\subsection{Object-Based Media}

Object-Based Media (OBM) is a method of breaking down film, television, radio or other media programmes semantically into smaller constituent chunks or objects. Relationships between these objects are also defined such that they can be recombined without losing their core meaning (for example narrative or functional role within a programme). In OBM terms all different parts of the media are objects, for example, audio-visual sequences, sound, music, and overlays (such as sign-language or subtitles). Simple uses of OBM include creating abridged versions of programming, allowing viewers to catch up on the salient material within several episodes in a short amount of time; personalised sound-level mixing; plot tweaks to feature content aligned to viewers' interests. The LRoTF utilises an OBM tool and takes the novel step of incorporating physical IoT objects from the viewing environment and coding them as media objects within the OBM. This allows the media to be reflected in the physical environment and allows the physical environment to effect influence the media.

\subsection{Related Approaches}

In addition to OBM the LRoTF builds up on related research exploring means to enhance immersion including Perceptive Media [1] and IoT Storytelling [2]. Classical Interactive Storytelling utilises audience input to influence storylines through direction action, as exemplified recently through 
Netflix's Black Mirror episode Bandersnatch ${ }^{1}$. Perceptive Media [1], contrastingly, uses information about audiences and sensor data to subtly alter media without any noticeable interaction with an audience. While story arcs remain the same for each viewing, aspects of the narrative are tweaked in order to create a more context-specific, situated cf. [3], experience for the audience. Similarly IoT Storytelling synchronises onscreen events with actuations from IoT devices acting in the viewer's context as the story progresses [2].

Although delivered by using technology, both IoT Storytelling and Perceptive Media are similar to non-technical approaches to immersion. For example, a traditional storyteller may adapt aspects of the story's interior world to the particularities of the location and the group of listeners and may increase audience immersion by referring to events happening in the real world, for example the current weather conditions. These ways of altering how the story is delivered, either by reacting to the audience's context or by altering their environment, we describe as 'diegetic ${ }^{2}$ influencers' [4].

LRoTF combines elements of these approaches in order to test immersion that transcends the divide between the world of the media and the viewing context. This novel approach differs from the majority of immersive approaches in that, rather than in a 'liminal' mode - forcing the viewing out of the real and into the virtual by harnessing technologies like high-fidelity screens or Virtual Reality - it operates in a 'liminoid' mode [4], subtly blurring the real/virtual boundary (Figure 1).

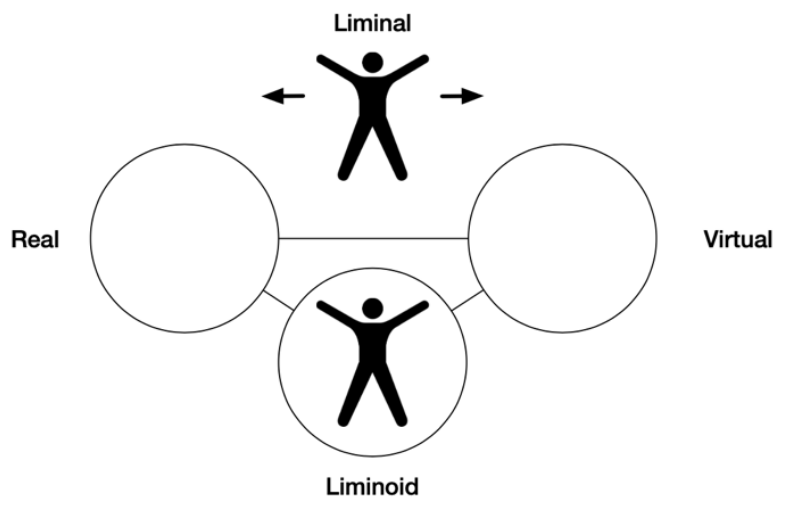

Figure 1. Liminal and liminoid perspectives on real and virtual.

\subsection{The Space}

As a technology demonstrator the LRoTF, as the name suggests, takes place in a living room. In order to enable its role as a demonstrator, however, it has had to be moveable and reconfigurable. Across various installations at Liverpool's Foundation for Art and Creative Technology, The Victoria \&

\footnotetext{
${ }^{1}$ Blackmirror: Bandersnatch is as an interactive film in which the viewer can make decisions for the main character, the young programmer Stefan who is adapting a fantasy choose-your-own-adventure novel into a video game. Viewers have ten seconds to make choices, or a default decision is made. Once a playthrough ends, the viewer is given an option of going back and
}

Albert Museum, The Buildings Research Establishment, Tate Modern Gallery, and Lancaster Institute for the Contemporary Arts, LRoTF creates the essence of living room, within the relevant space. A television screen, seating, lamp-based lighting, coffee table, and supporting living room accoutrements provide the basis for the demonstrator (Figure 2).

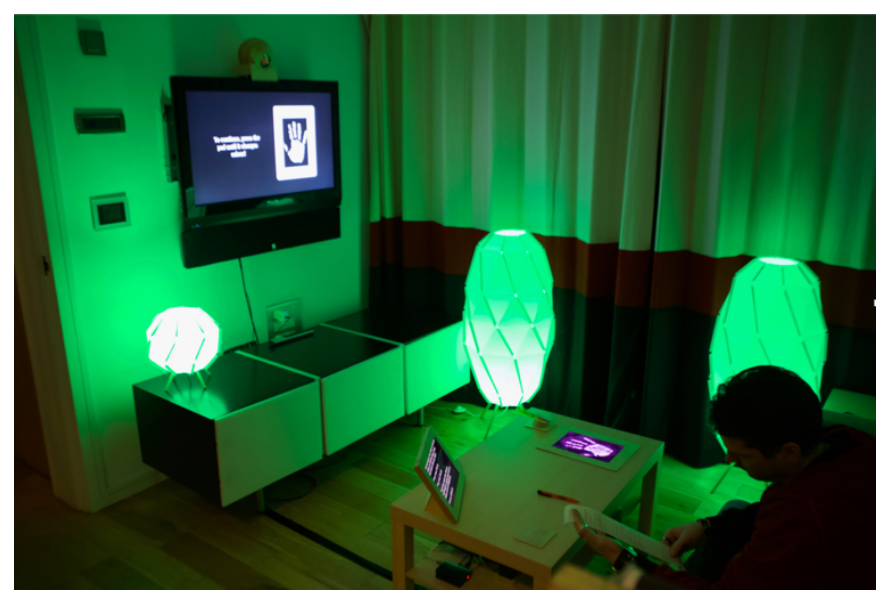

Figure 2. The Living Room Demonstrator.

\subsection{The (IoT) Objects}

A combination of off-the-shelf IoT devices and purpose made research prototypes make up the sensors and actuators within the room that enable the immersive experience. These include a smart lighting system, connected heating/cooling system, connected plug-sockets, automatic blinds, a thermal printing device, coffee-table-based tablet, posterior sensors in the seating, face-scanner, reactive coffee-table surface, and palmprint consent device. Some of the IoT objects played straightforward roles within the experience-for example the lights simply mirror the tone of video content, the automatic blinds punctuate the experience, and the heating/cooling system adds real wind in the room when there is wind on screen. Others are more subtly and provocative. The thermal printer gives audiences a hard-copy receipt of all their data interactions in the room and a means for them to provide signatory consent, while the face-scanner and palm-print consent device (although not functional) provide the illusion that collecting and processing biometric data are necessary and normal.

\subsection{The Voice}

LRoTF's primary means of communicating with the user is a voice interface. Using commercial text-to-speech software we created a persona for the living room that informs those experiencing the demonstrator what is happening throughout their experience. The living room's irreverent and uncompromising turn of phrase was useful as a tool to entertain audiences and to corral them into the necessary making a different choice. The average viewing is reportedly 90 minutes, though the quickest path ends after 40 minutes.

\footnotetext{
2 Diegesis is a style of storytelling that presents an interior view of a world in which: details about the world itself and the experiences of its characters
} are revealed explicitly through narrative. 
behaviours to access the full experience. A fascinating property of the demonstrator, however, is the obedience that audiences show in response to the voice's verbal requests. For example, when asked to hold up copies of their hard-copy consent forms and to smile for the camera, $100 \%$ of participants obliged without questioning what was happening.

\subsection{Consentr}

While earlier incarnations had used the voice interface to obtain consent for the (fictional) interactions in the living room, in order to create a self-sufficient version, the Consentr device was built. This is a palm-scanning device that, in essence, acts as a button. Users are prompted to place their hand on the device, which authenticates them by their palm print, at key points of the experience if they wish to proceed (Figure 3).

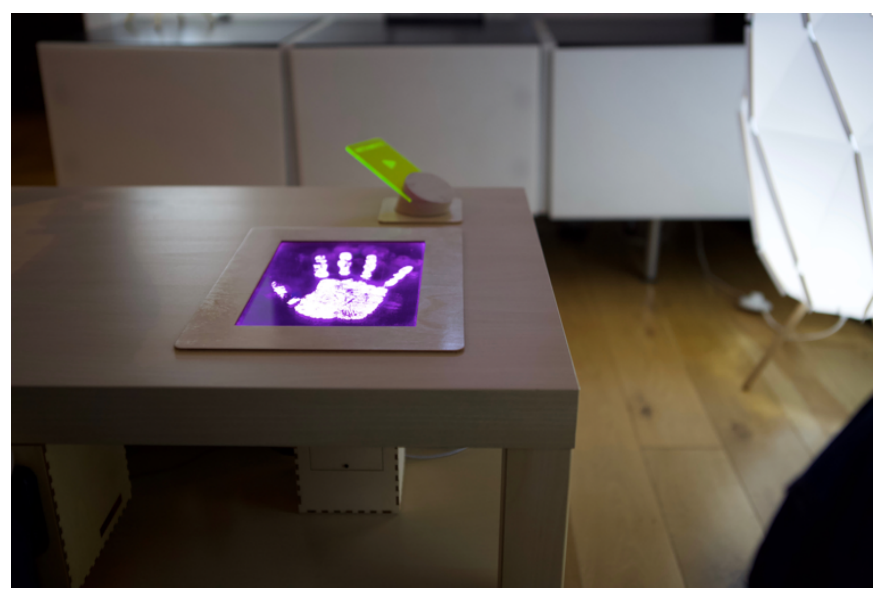

Figure 3. Consentr palm-scanning prototype.

\subsection{Media}

The LRoTF experience is based around a short drama titled The Break Up. This story, commissioned by BBC Research and Development, tells the story of an abusive relationship that has broken down. The two protagonists meet up in a café to attempt reconciliation. Through the use of OBM the story arc resolves in several different ways, and other aspects of the media (for example the use of colour grading) reflect the realworld environment. Taking the possibilities of OBM to the extreme, depending on context and audience reactions, The Break Up can be 'genre spiked' and take a narrative detour into an alternate sci-fi Universe that contains the same characters, but with the gender roles swapped and the relationship's challenges taking place across time and space. The Break Up was scripted, filmed, edited and implemented into the LRoTF in such a way that using OBM it can deliver a completely unique experience of the media to audience members.

\section{Futures}

\subsection{Methods Landscape and Design Fiction}

There is a gamut of methods used in order to try and apprehend insights about the future of technology, for example Horizon Scanning, Futurescaping, design ethnography, and critical design [5]. Among this methodological landscape Design Fiction has become a significant and flexible approach for exploring and understanding plausible futures [6]. Discussion about the precise meaning of Design Fiction are ongoing - "Its meaning has remained somewhat up for grabs within the research community" [7]. Bleecker's early observations include a striking argument describing how fact and fiction are all tangled up anyway, so if we deliberately create fictions we can certainly influence fact [8]. Subsequent and more nuanced discussions describe how Design Fictions create the opportunity for meaningful discussions about futures by building fictional worlds [9] and those worlds are built by creating several artefacts that each provide "entry points" to their interiors [10]. In the parlance of Design Fiction the interior of the fictional world - that is the future which is being explored - is referred to as the "diegesis" and the artefacts that make it up are "diegetic prototypes" [11].

\subsection{Living the Diegesis: Experiential Design Fictions}

Traditionally Design Fiction methods create several artefacts that create a reciprocal prototyping loop; the artefacts test the quality of the fictional world and the world tests the quality of the artefacts. Together researchers can use this loop to develop rich insights [10]. The LRoTF works in the same way but builds the diegesis in such a way that anyone can actually experience it. Audiences (of the Design Fiction) can sit within the fictional world and interact with it. Effectively this envelope the audience within the diegesis, and they themselves become one of the diegetic prototypes that make it up. Building upon the theory that in Design Fictions "the format is the message" [12], this approach-where it is possible to achieve - can arguably immerse the Design Fiction's audience to the maximum possible degree, and thus open up the space for meaningful discussions in a uniquely powerful way. This approach to futures methodology is not completely unique, for example they are similar to Candy's the roleplaying elements of Candy's Experiential Futures [13] and Elsden et al.'s Speculative Enactments [14], however in the LRoTF the interactivity that supports the diegesis is enacted by the system rather than by humans. Although crafting such experiential Design Fiction is not always possible, and hence is not proposed as a universal approach for Design Fiction practitioners, in this paper we have described one circumstance where a Design Fiction can be experienced directly. In the following we reflect on the process of creating the LRoTF and describe our preliminary findings stemming from this example of an Experiential Design Fiction.

\section{Reflections on the Living Room Experience}

\subsection{Contexts of Study}

The LRoTF has been installed and used as a research probe in several different contexts. Various iterations of the project have been exhibited at the Foundation for Art and Creative 
Technology, the Victoria \& Albert Museum, Tate Modern, and Lancaster Institute for the Contemporary Arts. The context of the research described in this paper, however, relates to its installation as a technology demonstrator at the Buildings Research Establishment (BRE) — a world leading multi-disciplinary buildings science research centre. Throughout the lifetime of the project insights have been developed using a range of different epistemological stances including Anticipatory Ethnography [15],

ethnomethodological analysis of conversations [16], and Research through Design (Fiction) [17]. The following are based on elements of research derived from each research approach and reflect the current iteration of the LRoTF demonstrator.

\subsection{Revealing Complexity}

One purpose of the prototype was to reveal the complexity of the "constellations" [18] that make up many IoT use-cases in such a way that audiences would be "informed by design" [19]. Through different iterations this was approached in various ways. Initially the use of photoluminescent materials that would glow when exposed to Ultra Violet light, allowed us to highlight interconnectivity between objects in the room during the concluding moments of the experience. This sudden reveal and explicit communication of interconnectivity was juxtaposed against an otherwise seamless experience - the contrast intended as a device to highlight the complexity of interconnections. While effective, this approach was later augmented with the addition of a step-by-step consent procedure. First using the 'voice' of the room (and verbal feedback from participants) to lead audiences through a process whereby they agree to have their faces scanned and data feeds aggregated, and in later iterations utilising the Consentr hand pad, the incorporation of a GDPR-minded process that attempts to gain unambiguous consent revealed the complexity of the system in no uncertain terms. Throughout the experience a tablet placed on the coffee table and the thermal printer underscored this transparent exposition of complexity. Our assemblage of research approaches show that audiences respond positively to these attempts to design in transparency, seeing and understanding the extent to which data and devices interact with one another as well as acting independently.

Significantly, on occasions when audiences were confused or unclear as to the system's complexity, these efforts provided means to properly engage with the uncertainty.

\subsection{Compliance and Dissonance}

A fascinating facet of the experiences designed using the living's voice as a guide for audiences is that users, universally, complied with requests. In a handful of occasions these have been deliberately outlandish and tended toward pushing audiences into extreme positions. Nonetheless, whether being asked to smile so that a face scan can be combined with employment data and purchase history, to provide a hard copy signature agreeing to terms, and smiling for the camera as that signature is photographed by the system, audiences were extremely compliant. While consistent this compliance comes hand-in-hand with an observable dissonance between actions in the moment and post-hoc reflections; although proceeding without much thought for the consequences at the time of consent, audiences later suggested that they wouldn't really like to submit their consent for data gathering and processing under these circumstances. The impact of the LRoTF's (un)real nature on this dissonance is, as yet, unclear.

\subsection{Mixed Feelings on Perceptive Media}

Audiences reported mixed feelings on aspects of the LRoTF as a media experience and as a way of consuming television content. While aspects of the room's immersive technologies were generally well received - the adaptive lighting for example - the non-linearity of Perceptive Media used for television was an aspect that audiences tended to be sceptical about. It was clear that audiences were concerned about the impact on shared experiences. For example, shared 'secondscreen' experiences (e.g. reading and contributing to Twitter commentary on a programme whilst watching it) would be undermined by content that materially differed for different viewers. Similarly, sharing post-hoc conversations about programming was identified as an area that would potentially be detrimentally affected by such a system.

\subsection{Living with the Living Room of the Future}

As a demonstrator and provocation showcasing the potential uses of the IoT as a means to deliver 'liminoid' immersive media experiences LRoTF has been extremely well received. Through exposure at high profile cultural venues the demonstrator has reached large audiences $(400+)$ and facilitated a wealth of insightful conversations, some of which will be represented in supporting research outputs [4]. Aspects of the LRoTF are, as opposed to speculative visions of what might be, good representations of what is now-for example audience profiling in online streaming systems. Notwithstanding the similarity to systems that are widely adopted, the majority of audiences reacted to LRoTF's proposition with a small portion of distain: while they were almost always intrigued by the technology, they were usually not that keen on having such a system in their home.

\section{Conclusion}

The IoT will continue to impact how we live and work. Within those impacts are changes on how we locate, consume, and experience media. The LRoTF technology demonstrator shows how current and emerging technologies may come together and enhance the ways in which we experience media in our living rooms. In contrast to liminal approaches to immersion, the LRoTF's liminoid experience is more subtle 
and utilises the ambient technologies resulting from adoption of domestic IoT devices to create more subtle liminoid experiences. When combined with perceptive media and object-based media, such an approach provides the means to create and curate entirely new types of media experience, that, as well as delivering immersion in novel ways, may potentially disrupt and reconfigure our relationships with television, film, and radio. The LRoTF also highlights an emerging methodology that transmutes Design Fiction from passive objects toward dynamic and lived experiences. This 'experiential' means to research 'Implications for Adoption' [20] provides the blueprint for researchers to quickly-butmeaningfully demonstrate and prototype near future visions in order to stimulate rich conversations around emerging technologies.

\section{Acknowledgements}

The project has been made possible through the PETRAS Cybersecurity for the Internet of Things Research Hub under EPSRC grant EP/N02334X/1 and the Objects of Immersion research project under AHRC grant (AH/R008728/1) with additional support from the North West Consortium Doctoral Training Partnership, the British Council, BBC Research and Development, the Foundation for Art and Creative Technology, the Victoria and Albert Museum, and Tate Modern.

\section{References}

[1] A. Gradinar et al., "Perceptive Media - Adaptive Storytelling for Digital Broadcast," in Proceedings of INTERACT 2015, vol. 158, 2015, pp. 586-589.

[2] P. Coulton, "Sensing Atoms and Bits," in Sensory Arts and Design, I. Heywood, Ed. Bloomsbury, 2017, pp. 189-203.

[3] L. Suchman, Human-Machine Reconfigurations: Plans and Situated Actions. Cambridge: Cambridge University Press, 2007.

[4] P. Coulton et al., "Experiencing the Future Mundane," in Proceedings Research Through Design Conference, 2019.

[5] N. Raford, "From Design Fiction to Experiential Futures," in The Future of Futures, Houston, TX, USA: The Association of Professional Futurists, 2012.

[6] J. Lindley, "A thesis about design fiction," Lancaster University, 2018.

[7] J. Tanenbaum, "Design fictional interactions," Interactions, vol. 21, no. 5, pp. 22-23, Sep. 2014.

[8] J. Bleecker, "Design Fiction: A short essay on design, science, fact and fiction," Near Future Laboratory, 2009 .

[9] J. Lindley and P. Coulton, "Back to the future," in Proceedings of the 2015 British HCI Conference on -
British HCI '15, 2015, pp. 210-211.

[10] P. Coulton, J. Lindley, M. Sturdee, and M. Stead, "Design Fiction as World Building," in Proceedings of the 3rd Biennial Research Through Design Conference, 2017, pp. 163-179.

[11] D. Kirby, "The Future is Now: Diegetic Prototypes and the Role of Popular Films in Generating Realworld Technological Development," Social Studies of Science, vol. 40, no. 1, pp. 41-70, Sep. 2010.

[12] P. Coulton, J. Lindley, and H. Ali, "Design Fiction: Does the search for plausibility lead to deception?," in Proceedings of DRS 2016, Design Research Society 50th Anniversary Conference., 2016.

[13] S. Candy, "The Futures of Everyday Life: Politics and the Design of Experiential Scenarios," University of Hawai'i, 2010.

[14] C. Elsden et al., "On Speculative Enactments," Proceedings of the 2017 CHI Conference on Human Factors in Computing Systems, pp. 5386-5399, 2017.

[15] J. Lindley, D. Sharma, and R. Potts, "Anticipatory Ethnography: Design Fiction as an Input to Design Ethnography," Ethnographic Praxis in Industry Conference Proceedings, vol. 2014, no. 1, pp. 237253, Oct. 2014.

[16] A. Crabtree, M. Rouncefield, and P. Tolmie, Doing Design Ethnography. London: Springer-Verlag, 2012.

[17] J. Lindley, "A pragmatics framework for design fiction," in Proceedings of the European Academy of Design Conference, 2015.

[18] J. Lindley, P. Coulton, and R. Cooper, "Why the Internet of Things needs Object Orientated Ontology," The Design Journal, vol. 20, no. sup1, pp. S2846-S2857, Jul. 2017.

[19] J. Lindley, P. Coulton, and R. Cooper, "Informed by Design," in Living in the Internet of Things: PETRAS Conference, 2018.

[20] J. Lindley, P. Coulton, and M. Sturdee, "Implications for Adoption," in Proceedings of the 2017 CHI Conference on Human Factors in Computing Systems - $C H I ' 17,2017$, pp. 265-277. 
